Voix et Images

voixetimages

\title{
La Maison rouge du bord de mer de Hugues Corriveau : la blessure du désir
}

\section{Claude Sabourin}

Volume 18, numéro 1 (52), automne 1992

Les écritures masculines

URI : https://id.erudit.org/iderudit/201008ar

DOI : https://doi.org/10.7202/201008ar

Aller au sommaire du numéro

Éditeur(s)

Université du Québec à Montréal

ISSN

0318-9201 (imprimé)

1705-933X (numérique)

Découvrir la revue

Citer cet article

Sabourin, C. (1992). La Maison rouge du bord de mer de Hugues Corriveau : la blessure du désir. Voix et Images, 18(1), 162-166.

https://doi.org/10.7202/201008ar d'utilisation que vous pouvez consulter en ligne.

https://apropos.erudit.org/fr/usagers/politique-dutilisation/ 
Roman

\section{La Maison rouge du bord de mer de Hugues Corriveau: la blessure du désir}

Claude Sabourin, Université du Québec à Montréal

Avec son dernier roman ${ }^{1}$, Hugues Corriveau apporte une contribution magistrale au répertoire de la littérature érotique. Arrivé discrètement sur la scène littéraire à la fin des années 1970, l'auteur qui lançait Les Compléments directs aux Herbes rouges en 1978 bénéficie aujourd'hui de son expérience propre et de celle d'écrivains de la "génération - du texte, du corps, du sexe et du désir, issue elle-même d'écoles et de courants divers, tous centrés, les uns comme les autres "telqueliens * ou barthésiens, "underground. ou contre-culturels, minimalistes, : illisibles", etc., sur le ludisme verbal, la recherche de formes nouvelles et de modes d'expression éclatés comme moyens pour s'approprier d'abord le pouvoir et le pays (env. 1965-1972), le corps (chez 
les féministes, surtout, autour des années 1975) puis, plus près de nous, le "réel ", la " réalité" via la fiction, tels qu'en ont fait foi quelques membres et collaborateurs de La Nouvelle Barre du jour lors du Deuxième Colloque bissextile du 29 février 1984. Il y a donc loin entre les écrits de l'expérience extatique d'un Louis Geoffroy, d'un Emmanuel Cocke, d'un Paul Chamberland, d'un Patrick Straram, d'un Denis Vanier (pour ne nommer qu'eux) et ceux d'un Corriveau, préoccupé par une "modernité [qui] devrait s'occuper du réel ${ }^{2}$, c'est-à-dire de la mort qui se vit droit devant, de l'angoisse au quotidien. Mais il y aurait cependant proximité avec le regard que livrent les écritures de femmes sur le monde 3 . Bref, beaucoup d'encre a coulé depuis que "la modernité. a pris l'habitude de se renouveler. À l'heure actuelle, Corriveau ne pourrait s'inscrire en faux contre le "retour à Dieu " et une certaine "lisibilité,, tels qu'il les a pointés: La Maison rouge du bord de mer, éminemment "lisible", d'un propos fort recueilli, tout autant que peut l'être le Cantique des cantiques, unit ses thèmes de prédilection (amour, désir, blessure et mort) à ce contre quoi il s'élevait dans "Vouloir la fiction" (La Nouvelle Barre du jour, $\mathrm{n}^{\circ}$ 141). Comme si la chair et l'érotisme n'incluaient pas eux-mêmes leur propre part de mysticisme!

Son dernier titre tire grand parti du meilleur des romans et nouvelles de Le Clézio (Le Procès-verbal, Désert, La Ronde et autres faits divers). Ses deux adolescents, Ismïa et Yachar, tous deux âgés de douze ans, rappellent en effet les personnages de l'écrivain niçois. Comme eux ils sont en dérive, au bout de tout. Ils fuient la promiscuité des villes, la vie familiale et ses obligations, le travail qui les dévorent trop tôt. Ils démontrent cette même profondeur de caractère, une curiosité similaire quant à l'allure du monde, quant aux ravages du temps, quant à la conscience de soi et à la découverte de l'autre. L'initiation sexuelle d'Ismia et de Yachar prend alors tout son sens dans cette double révolution qu'est, pour chacun d'eux, la quête intérieure et le rapprochement mutuel; là s'établit la différence entre la pornographie et l'érotisme.

Ce que les jeunes protagonistes cherchent peut-être, c'est l'union de leurs corps, la douceur de l'amour, de la tendresse et du partage. Au terme de quarante-huit heures d'errance sur la côte presque déserte d'un pays d'Afrique du Nord, ils auront découvert le bonheur sexuel, certes - et cela à travers les tabous, la crainte, l'interdit, le geste intuitif, le discours tout réservé de leurs aînés en la matière -, mais aussi l'horreur des corps qui se mutilent par amour, témoins qu'ils sont de scènes sadomasochistes que donnent volontairement à voir Julia et Czeslaw, locataires de la maison rouge du bord de mer, et d'ébats ludiques, à la limite de la violence, auxquels s'adonnent les 
jumeaux homosexuels de la baraque sur la dune. De retour en milieu urbain, après qu'ils eurent *tout* vu, s'insinue en Ismïa et Yachar le goût du sang et de la douleur qui les mènera à réaliser la fantaisie érotique de la blessure. Fantasme qu'ils ne s'expliquent que par la crainte de voir, chez soi comme chez l'autre, le désir se banaliser.

La réussite de Corriveau tient à l'élaboration lente et patiente d'un espace sensuel (voir, toucher, sentir, goûter sont les verbes les plus récurrents) et à la narration itérative où se resserre la description de scènes érotiques invariablemement mises en alternance avec une pléthore d'images relatives à la faune (aviaire; surtout), la flore et l'environnement africains. Aussi, les changements de perspectives (trait typique de la littérature érotique) assurent au roman (16 chapitres de 9 à 10 pages chacun) l'efficacité nécessaire contre toute lassitude: tantôt le lecteur bénéficie d'une description immédiate, celle du narrateur, tantôt il admire les corps enlacés à travers le regard intrigué d'Ismiia et de Yachar. Eux-mêmes curieux l'un de l'autre, ils s'interrogent à tour de rôle sur leur propre venue à la sexualité avant leur union. La narration à relais emplit alors ses fonctions quant à la diversification des points de vue et des scénarios. Ainsi en est-il des récits intercalés d'Ismïa et de Yachar qui évoquent en toute franchise et de plein gré les plaisirs sexuels que léur sœur et frère aînés leur ont procurés comme initiation à la vie. Inceste homosexuel épanouissant pour chacun d'eux, contrairement à son caractère coupable dans $\mathrm{Du}$ masculin singulier et Les Taches de naissance, mais dont les aboutissants diffèrent. Tandis que Rachel allume chez Ismïa l'onirisme de l'ibis bleu (orgasme, amour et tendresse conjugués), Yacheb, lui, offre à son frère la satisfaction d'un devenirhomme: l'abandon au désir de son partenaire pour jouir de ce dernier en retour, jusqu'à la perte du souffle. Mais pour Ismïa l'accès à la féminité - résidu culturel de son Afrique natale? - ne saura véritablement se jouer que par le sang, lors de la rupture de l'hymen, moment que Yachar craint viscéralement, autant pour sa partenaire que pour luimême; ce sera d'ailleurs sa compagne qui s'empalera sur lui afin que coule ce sang unique de la femme, que laveront les eaux de la mer, selon le rituel. Bref, sur le plan formel, Corriveau, classique, procède par addition, surenchère et variation, tel qu'en général le genre le prescrit, si l'on accepte, évidemment, dès l'ouverture du roman, l'auto-érotisme comme le premier degré d'excitation que l'on abandonne au lecteur.

Voyage initiatique donc dans le dómaine "étrange de la sexualité adulte que celui d'Ismia et de Yachar! Inoubliables moments de communion avec le corps qu'amène la rencontre des deux fugueurs. Mais que quarante-huit heures pour tout assimiler ce que leur jeunesse ignore encore des plaisirs charnels! Combien de fois l'auteur soulève 
leur trouble, leur bouleversement... et leur fascination? Ils sont aspirés par un désir inassouvi de découvertes, fascinés par cette violence démente des jeux sexuels qui sourd de la maison rouge, notamment, et qui transpire à plein dans les personnages de Julia et Czeslaw, " oiseaux de proie - qu'ils ne demandent qu'à intégrer à leurs fantaisies érotiques la chair fraîche. Justement, ces 48 heures - tout voir et faire tant en si peu! - et les douze ans des Ismïa et Yachar pourraient miner le crédit du roman. Pourtant, il n'en est rien. Les adolescents atteignent cette maturité physique et psychique propre à les porter jusqu'à l'expérience de la déchirure, de la sensualité vécue jusqu'au plus profond de l'être sans que l'on en ressorte choqué ou dubitatif. Habileté de l'écrivain, qu'il faut suivre là où il se trouve depuis ses débuts (la psychologie des profondeurs), mais aussi choix d'un contexte ethnographique en cohésion avec les projections de l'imaginaire occidental à l'égard des mœurs sexuelles nord-africaines, censément hâtives, non sélectives quant au sexe du partenaire, souvent vénales. Ajoutons à cela que le temps des Le Clézio, comme des Ben Jelloun - de qui Corriveau s'inspire également - relève de la ponctuation astrale: aube, midi, minuit, lune, soleil, étoiles, marées, etc. L'aventure d'Ismïa et de Yachar semble alors se dérouler dans un hors-temps, dans un espace temporel dilaté (mais dont la mesure n'a certes pas le faible coefficient d'une folle échappée de week-end) qu'une application adéquate des modes verbaux se plaît à laisser être.

En ce qui touche l'écriture donc, et les procédés littéraires, tout un monde sépare le Corriveau des Compléments directs de celui d'Autour des gares (L'Instant même, 1991). La "volte-face " attribuable jusqu'à un certain point à son intérêt pour les écritures de femmes (*je continue à [les] entendre comme une leçon ${ }^{4}$ ), qui rendent *mieux actuellement [la modernité] ${ }^{5}$ *, se situe à proximité, aussi, de l'initiative d'un de Bellefeuille, de qui Corriveau dit se sentir près ${ }^{6}$, tenté qu'il fut par l'expérience de la clinéarité - dans Ce que disait Alice (L'Instant même, 1989). Quelques attitudes narratives et traits de style, tout comme le souffle, se sont effectivement modifiés; la superposition des récits, par exemple, devient juxtaposition de scènes bien distinctes; les tours nominaux fréquents, en guise de phrases autonomes, sont presque désormais délaissés, tout autant que les propositions verbales centrées sur l'infinitif et les compléments du verbe hachurés par le point mode des : modernes" qu'aurait vraisemblablement introduite AnneMarie Alonzo; les subordonnées trouvent maintenant leur(s) principale(s), etc. Or, dans son apparente - platitude - stylistique et narratologique, La Maison rouge du bord de mer n'y perd pas en profondeur. L'écrivain garde sa griffe; il étoffe ses écrits du temps des Herbes 
rouges, qui n'ont plus désormais l'allure que d'avant-textes, d'ébauches, d'écrits d'une époque où surtout l'on se formait le style sous les couverts de la "modernité". Car il reste de Corriveau ce qui devait rester: l'angoisse individuelle - universelle - de la perte, ouverte au lecteur, et qui s'incarne, cette fois, dans une Afrique de détresse et de beauté.

1. Hugues Corriveau, La Maison rouge du bord de mer, Montréal, XYZ éditeur, coll. -Romanichels ., 1992, 162 p.

2. Id., -En avant... l'écrinure., La Nouvelle Barre du jour, septembre 1984, n 141, p. 30.

3. Ibid., p. 31.

4. Ibid.

5. Ibid., p. 30.

6. Ibid. 